\title{
Cardiovascular disease risk factors, depression symptoms and antidepressant medicine use in the Look AHEAD (Action for Health in Diabetes) clinical trial of weight loss in diabetes
}

\author{
R. R. Rubin, \\ Department of Medicine, The Johns Hopkins University School of Medicine, 600 North Wolfe \\ Street, Baltimore, MD 21287, USA. Department of Pediatrics, The Johns Hopkins University \\ School of Medicine, Baltimore, MD, USA
}

\section{S. A. Gaussoin,}

Department of Biostatistical Sciences, Wake Forest University School of Medicine, WinstonSalem, NC, USA

\section{Peyrot,}

Department of Medicine, The Johns Hopkins University School of Medicine, 600 North Wolfe Street, Baltimore, MD 21287, USA. Department of Sociology, Loyola University Maryland, Baltimore, MD, USA

\section{DiLillo,}

Department of Psychology, Ohio Wesleyan University, Delaware, OH, USA

K. Miller, Department of Education, University of North Carolina, Chapel Hill, NC, USA

T. A. Wadden, Department of Psychiatry, University of Pennsylvania, Philadelphia, PA, USA

D. S. West,

College of Public Health, University of Arkansas for Medical Sciences, Little Rock, AR, USA

\section{R. R. Wing, and \\ Department of Psychiatry and Human Behavior, Brown University, Providence, RI, USA}

\section{W. C. Knowler}

Diabetes Epidemiology and Clinical Research Section, National Institute of Diabetes and Digestive and Kidney Diseases, Phoenix, AZ, USA

for the Look AHEAD Research Group

R. R. Rubin: rrubin4@jhmi.edu

\author{
Abstract \\ (C) Springer-Verlag 2010 \\ Correspondence to: R. R. Rubin, rrubin 4 @ jhmi . edu. \\ Electronic supplementary material The online version of this article (doi:10.1007/s00125-010-1765-1) gives details of the Look \\ AHEAD Research Group at baseline, which is available to authorized users. \\ Duality of interest The authors declare that there is no duality of interest associated with this manuscript. \\ Trial registration Clinicaltrials.gov NCT00017953
}


Aims/hypothesis-To determine the associations of baseline depression symptoms and use of antidepressant medicines (ADMs) with baseline cardiovascular disease (CVD) risk factors in Look AHEAD (Action for Health in Diabetes) trial participants.

Methods-Look AHEAD participants $\left(n=5,145\right.$; age $58.7 \pm 6.8$ years; BMI $\left.35.8 \pm 5.8 \mathrm{~kg} / \mathrm{m}^{2}\right)$ were assessed for CVD risk factors (elevated $\mathrm{HbA}_{1 \mathrm{c}}$ or insulin use, elevated $\mathrm{BP}$ or antihypertensive use, elevated lipid levels or lipid-lowering medication, current smoking, BMI $\geq 30 \mathrm{~kg} / \mathrm{m}^{2}$, lower peak exercise capacity assessed as metabolic equivalents [METs], and ankle-brachial index $<0.9$ or $>1.3$ ). Participants also completed the Beck Depression Inventory (BDI) and reported their use of ADMs.

Results-Of the participants, $14.7 \%$ had BDI scores $\geq 11$, consistent with mild-moderate depression, and $16.5 \%$ took ADMs; $4.4 \%$ had both depression markers (i.e. elevated symptom scores and took ADMs). In logistic regression analyses of CVD risk (elevated risk factor or use of medication to control the risk factor), controlled for demographic factors, continuous BDI scores and ADM use were each independently associated with elevated BP (or medication), current smoking, BMI $\geq 30 \mathrm{~kg} / \mathrm{m}^{2}$ and lower MET values. ADM use was also associated with elevated serum lipids or use of lipid-lowering medication.

Conclusions/interpretation-Among Look AHEAD participants, depression symptoms or ADM use on entry to the study were each independently associated with a wide range of CVD risk factors. Future research should assess the temporal dynamics of the relationships of depression symptoms and ADM use with CVD risk factors.

\section{Keywords}

Antidepressant medication; CVD risk; Depression symptoms; Look AHEAD trial

\section{Introduction}

Rates of cardiovascular disease (CVD) and CVD mortality are disproportionately high in people with diabetes [1]. This fact may be explained in part by the disproportionately high rates of depression in this population [2], since depression is associated with adverse CVD outcomes [3,4]. Mechanisms underlying the associations among diabetes, depression and CVD have not been determined; while many suggestions have been made, perhaps the most straightforward is that depression is linked to a variety of known CVD risk factors, e.g. physiological risk factors (glucose, BP, lipids and obesity), as well as behaviours such as cigarette smoking, decreased physical activity and poor diet.

It is also unclear whether the use of antidepressant medicines (ADMs) affects CVD risk. Tricyclic antidepressants (TCAs) and monoamine oxidase inhibitors affect cardiac conduction and rhythm, and cardiotoxic side effects of these medicines have been reported [5]. Use of newer, more commonly prescribed ADMs, such as selective serotonin reuptake inhibitors (SSRIs) and serotonin-noradrenaline (norepinephrine) reuptake inhibitors (SNRIs), may reduce platelet aggregation and improve cardiovascular morbidity and mortality in patients with established CVD [5]. However, these benefits have not been found in all studies, and they appear to be minimal when they are present $[6,7]$.

The possibility that ADM usage might be associated with negative health outcomes was raised by findings from the Diabetes Prevention Program (DPP), which showed that participants in the placebo and intensive lifestyle intervention (ILI) arms of the study were two to three times more likely to develop diabetes during the course of the study if they were taking ADMs than if they were not [8]. The risk of developing diabetes in the DPP metformin group was not elevated among those taking ADMs. The possible health 
consequences of ADM use in people with type 2 diabetes deserve further study—if people with diabetes take ADMs at the same rate as the general population (15\%), approximately three million people in the USA who have type 2 diabetes are taking ADMs.

To date there have been few, if any, studies on the association of depression symptoms or ADM use with CVD risk in people with type 2 diabetes, or studies-among people with diabetes or in the general population - that simultaneously assess the independent association of depression symptoms and ADM use with CVD risk.

The Look AHEAD (Action for Health in Diabetes) clinical trial offers an opportunity to assess the independent association of depression symptoms and ADM use with CVD-related behavioural and clinical risk factors (smoking, fitness and BMI, and blood glucose, lipids and BP), controlled for potential confounders. Based on the existing literature we hypothesise a significant positive association between depression symptoms and CVD risk factors in the Look AHEAD participants, as well as a significant positive association between ADM use and CVD risk factors. Moreover, we hypothesise that these associations will be additive, such that neither association is contingent upon the other. Since this is a cross-sectional study we cannot draw conclusions about causal associations between depression measures and CVD risk measures, but the results of this study can generate hypotheses to be tested in future longitudinal studies.

\section{Methods}

The primary objective of the Look AHEAD trial is to assess the long-term effect (up to 14 years) on cardiovascular and other health outcomes of a comprehensive behavioural weight loss intervention in a randomised clinical trial of 5,145 overweight or obese individuals with type 2 diabetes. Participants were randomised to an ILI or to a usual care intervention, referred to as diabetes support and education. Participants are being followed at 16 clinical centres in the USA. On entry to the study Look AHEAD trial participants were $45-76$ years of age (which was changed to 55-76 years during the second year of recruitment to increase the anticipated cardiovascular event rate). Participants had a BMI $>25 \mathrm{~kg} / \mathrm{m}^{2}\left(27 \mathrm{~kg} / \mathrm{m}^{2}\right.$ if currently taking insulin, because insulin use is known to increase weight), $\mathrm{HbA}_{1 \mathrm{c}}<11 \%$, systolic BP $<160 \mathrm{mmHg}$ and diastolic $<100 \mathrm{mmHg}$ and triacylglycerols $<6.78 \mathrm{mmol} / \mathrm{l}$. Potential participants were excluded if they had underlying diseases likely to affect the safety of the interventions or factors that might limit adherence to the interventions or affect conduct of the trial, including hospitalisation for depression in the past 6 months, suicidal ideation, current diagnosis of schizophrenia, other psychotic disorders or bipolar disorder, self-report of alcohol or substance abuse within the past 12 months, or other medical, psychiatric or behavioural limitations (e.g. difficulty completing the 2 week run-in period during which participants were required to record food eaten) that in the judgement of the principal investigator might interfere with study participation or the ability to follow the protocol. ADM use and depression symptoms that did not require hospitalisation in the prior 6 months and did not involve suicidal ideation were not criteria for exclusion. Full details of the Look AHEAD design and methods are reported elsewhere [9]; however, measures relevant to this report are briefly described below.

\section{Assessments}

Anthropometry-At the beginning of the study, all participants were assessed for weight, height, BP and ankle-brachial index (ABI). Weight and height were assessed in duplicate using a digital scale and a standard stadiometer. Seated BP was also measured in duplicate using an automated device after a $5 \mathrm{~min}$ rest. Leg-specific ABI was calculated according to a standard algorithm reported elsewhere [10]. 
Fitness-Participants completed a maximal graded exercise test to assess fitness before randomisation. Fitness was assessed using a standardised maximum graded exercise test and expressed as metabolic equivalents (METs): 1.0 MET is approximately $3.5 \mathrm{ml}$ oxygen uptake (kg body weight) ${ }^{-1} \mathrm{~min}^{-1}$. Details of the test were reported earlier [11].

Serum measurements-Standardised analyses of frozen specimens were conducted at the Central Biochemistry Laboratory (Northwest Lipid Research Laboratories, University of Washington, Seattle, WA, USA). Details of $\mathrm{HbA}_{1 \mathrm{c}}$, fasting serum glucose, total serum cholesterol, LDL-cholesterol, HDL-cholesterol and triacylglycerol measurement techniques were reported earlier [12].

Medical history-History of CVD was based on self-reported myocardial infarction, stroke, transient ischaemic event, percutaneous transluminal coronary angioplasty or coronary artery bypass graft. All participants reported whether they were current, past or never smokers.

Depression symptoms and ADM use-Current use of ADMs was assessed when participants brought all prescription medicines to their randomisation visit. On entry to the study, participants completed the Beck Depression Inventory (BDI) [13], a scale with reliable psychometric characteristics across a broad spectrum of both clinical and nonclinical populations. The BDI lists 21 symptoms, with responses scored from 0 to 3 in ascending symptom severity, and total scores ranging from 0 to 63 . We identified participants with BDI scores $\geq 11$ as having elevated depression symptoms; others have chosen scores ranging from 10 to 16 , generally as a function of the importance placed on depression recognition. The cut-off point of 11 was used in the analysis of depression symptoms in the DPP [14], allowing for direct comparison across the two study populations.

When assessing the association between depression symptoms and CVD risk, we used continuous BDI scores, as preliminary analyses indicated that dichotomising BDI scores introduced measurement imprecision that attenuated the associations between depression symptom scores and CVD risk, and because we found no non-linear associations between depression symptom scores and CVD risk (results not shown). To permit a more meaningful interpretation for the association of depression symptoms and CVD risk, the ORs were calculated based on an accepted criterion for a 'minimal detectable difference' [15] in BDI scores: $0.5 \mathrm{SD}$ of the baseline BDI value. In the current study $0.5 \mathrm{SD}=2.484 \mathrm{BDI}$ score points. This transformation makes the OR for BDI (the increase in odds of CVD risk associated with a one-point increase in the recalculated symptom measure) interpretable as the increase in CVD risk associated with a minimal detectable difference in BDI symptoms.

Risk factor classification-A primary objective of the study was to assess the association between cardiovascular risk factors and elevated depression symptoms or ADM use. We considered current smokers, those with BMI $\geq 30 \mathrm{~kg} / \mathrm{m}^{2}$, and those with $\mathrm{ABI}<0.9$ or $>1.3$ to be 'at risk'. Both low and high ABI values have been associated with elevated CVD risk [16]. We also considered to be 'at risk' those participants who had $\mathrm{HbA}_{1 \mathrm{c}}$, $\mathrm{BP}$ and lipid values above treatment targets recommended by the ADA [17] or the Expert Panel on Detection, Evaluation, and Treatment of High Blood Cholesterol in Adults [18], or who were taking medicine to achieve these targets $[19,20]$. Taking medicine for a risk factor was regarded as an indicator of the presence of that risk factor, even if that risk factor was controlled by the medication.

Insulin was the only glucose-lowering agent considered a risk factor because almost all participants were taking some glucose-lowering medicine. All antihypertensive and all lipidlowering agents were considered as risk factors. We also defined a composite CVD risk 
score for each person as the number of risk factors present (of the total of 11 shown later in Table 2). This composite measure was chosen because standard models for assessing total CVD risk do not identify as risk factors those conditions that are controlled by medication (because the impact of a controlled risk factor on CVD outcomes is presumed to be minimal), whereas we regard these risk factors as outcomes in their own right regardless of whether they can be controlled by medication.

The association between depression measures and MET values was assessed with MET values as a continuous variable, because there appear to be no established cut-off points for defining risk status for this factor. Thus, MET values were not included in the composite measure.

\section{Statistical analysis}

Analyses included all randomised participants and were performed using SAS version 9.2 (SAS Institute, Cary, NC, USA). Bivariate relationships between ADM use and elevated BDI scores and age, sex, race and education were assessed using $\chi^{2}$ tests and multivariate logistic regression. We assessed the association of CVD risk measures with BDI scores and ADM use in three ways. First, we modelled dichotomous cardiovascular risk factors using multivariate logistic regression to determine their associations with ADM use and continuous BDI scores. Second, we modelled MET values using sex-specific multivariate regression models, described in a previous report [10], to determine the association with continuous BDI scores and ADM use. Third, we modelled the number of risk factors for each individual (composite risk score, controlled for age, race/ethnicity, education, diabetes duration and history of CVD) using Poisson regression (which is appropriate for measures that count numbers of events).

We also performed a series of ancillary analyses for each of the three risk measures described above (dichotomous risk factors, MET values and composite risk score). First, we assessed whether there was a statistically significant interaction between depression symptoms and ADM use, controlled for all other factors, to determine whether associations were additive. Second, we conducted all regression analyses within the two BDI strata (BDI $<11, \geq 11)$ to see if the relationship between continuous BDI score and CVD risk factors differed in those with low depression symptoms and those with more symptoms of depression. Third, we repeated all analyses with $\log _{e}$-transformed BDI scores, because BDI scores were not normally distributed (most participants had very low scores). Fourth, we tested for an age interaction with BDI scores and ADM use, because participants as young as 45 years old were included only during the first year of recruitment, with the lower limit changing to 55 years thereafter.

Finally, we repeated regression analyses, first including only those participants who were taking SSRI agents, and then including all participants who were taking any agent other than a TCA or tetracyclic agent. These analyses examined whether the associations between SSRI (or any non-TCA or tetracyclic) use and CVD risk factors differed from the associations between all ADMs combined and CVD risk factors.

Values are mean \pm SD unless stated otherwise.

\section{Results}

\section{Depression scores and ADM use at baseline}

Table 1 shows that on entering the study $16.5 \%$ of participants were taking ADMs and $14.7 \%$ had BDI scores $\geq 11$, probably indicating mild to moderate depression. More than a quarter of participants (26.8\%) had at least one of the two depression markers. Of the 
participants taking ADMs, 57\% were taking SSRIs, 24\% were taking SNRIs and 19\% were taking TCAs or tetracyclic agents. Depression symptom scores were mostly in the subclinical range (median $=8,25$ th percentile $=6,75$ th percentile $=9$ ) in part because many with severe depression symptoms resulting in hospitalisation or inability to successfully complete the run-in period were excluded from Look AHEAD participation. Elevated BDI scores and ADM use were more common among participants who were younger, female or less educated. Racial/ethnic differences in BDI scores and ADM use were also apparent, with more American Indians and fewer whites having BDI scores $\geq 11$ and more whites and fewer African-Americans reporting use of ADMs (Table 1).

\section{Depression symptoms, ADM use and CVD risk factors}

Table 2 shows the proportion of participants defined as 'at risk' for each CVD risk factor, with 'at risk' defined as having an elevated level or taking medicine for the risk factor.

Table 2 shows that continuous BDI scores were significantly associated with all measures of $\mathrm{BP}$ risk, current smoking and BMI $\geq 30 \mathrm{~kg} / \mathrm{m}^{2}$. Use of ADMs was significantly associated with all indicators of CVD risk, except for $\mathrm{HbA}_{1 \mathrm{c}} \geq 7.0 \%$ or insulin use and out-of-target $A B I$ values. In multivariate regression analyses of the association between depression indicators and MET values (results not shown), BDI scores were negatively associated with MET values for women $(\beta[\mathrm{SE}]=-0.011[0.005], p<0.05)$ and for men $(\beta[\mathrm{SE}]=-0.027$ [0.009], $p<0.05)$, and ADM use was negatively associated with MET values in women $(\beta$ $[\mathrm{SE}]=-0.244[0.067], p<0.001)$; in men the association was nearly identical $(\beta[\mathrm{SE}]=$ -0.232 [0.124], $p=0.06$ ), but not statistically significant in this smaller group (owing to the different covariates in the sex-specific models, we did not test for differences in coefficients). The mean number of CVD risk factors (of 11) for which an individual participant was at risk was $7.15(\mathrm{SD}=1.92 ;$ minimum $=0$, median $=8$, maximum $=11)$. Modelling the number of risk factors using Poisson regression controlled for age, sex, race/ ethnicity, diabetes duration and history of CVD (results not shown), there was a significant association with BDI score $(p=0.0146)$ and with ADM use $(p=0.0003)$.

We also conducted a series of ancillary analyses for each of the three risk measures described above (dichotomous risk factors, MET values and composite risk score), and the results confirmed the robustness of our original models (results not shown). First, there was no statistically significant interaction between depression symptoms and ADM use for any dichotomous risk variable nor for the composite risk score variable. When we added an interaction term for depression symptoms and ADM use to the models of MET values for men and women, both of the interactions were statistically significant $(p<0.05)$. In each case, the association of MET values with each depression factor was stronger (more negative) in the relative or absolute absence of the other depression factor.

Second, when stratified by BDI score $(<11, \geq 11)$, associations of BDI scores with CVD risk factors in each group were similar to those of the entire study population (results not shown). BDI score was significantly associated with all measures of BP in both BDI score groups. In the stratified analysis, BDI score was associated with current smoking only in the high BDI score group, and with BMI $\geq 30 \mathrm{~kg} / \mathrm{m}^{2}$ only in the low BDI score group.

Third, when we re-ran the models with log-transformed BDI scores we found similar relationships and almost identical ORs and 95\% CIs. Fourth, when we tested for an age interaction with BDI scores and ADM use we found only one significant interaction (BDI score and age, with HDL-cholesterol as an outcome; $p=0.05$ ). We then re-ran the original model for HDL-cholesterol, including only those participants 55-76 years old; this analysis generated the same relationships reported in Table 2. 
Finally, when we repeated the regression analyses (results not shown), first including only those participants who were taking SSRI agents, and then including all participants who were taking any agent other than a TCA or tetracyclic agent, the results were nearly identical to analyses involving individuals taking any ADMs.

\section{Discussion}

We hypothesised a significant positive association between depression symptoms and CVD risk. While we found no such associations for elevated depression symptoms (BDI scores $\geq 11$ ), we did find that a continuous measure of depression symptoms was significantly associated with all indicators of hypertension, and with current smoking, BMI $\geq 30 \mathrm{~kg} / \mathrm{m}^{2}$, higher composite risk scores and lower MET values. This suggests that a continuous measure may be a better way of conceptualising the association of depression symptoms and CVD risk indicators, especially since we found no non-linear associations between CVD risk indicators and depression symptom scores. These findings are consistent with studies showing that continuous depression symptom severity scores are better predictors of many diabetes self-care behaviours than categorically defined probable major depression [21]; and that sub-syndromal depression is associated with cardiac mortality [22]. We also found that the associations between BDI score and CVD risk factors were similar for those with higher and lower BDI scores. This suggests that there may be benefit in reducing depression scores no matter where one is in the distribution of scores-lower is better regardless of one's score.

We hypothesised a significant positive association between ADM use and greater CVD risk, and found many significant associations: ADM use was significantly associated with all indicators of hypertension and hyperlipidaemia, and with current smoking, BMI $\geq 30 \mathrm{~kg} / \mathrm{m}^{2}$, higher composite risk scores and lower MET values (i.e. with every risk factor except for $\mathrm{HbA}_{1 \mathrm{c}} \geq 7.0 \%$ [or insulin use] and out-of-target ABI values).

The fact that our composite index of CVD risk (the number of factors on which a study participant was at risk) was also significantly associated with both BDI score and ADM use suggests that the risk is cumulative or global rather than merely associated with different specific risk factors in different individuals.

Our ancillary analysis findings confirm the robustness of our original models. We found that the associations of depression symptoms and ADM use generally were independent of one another; there was a significant interaction in the association of depression symptoms and ADM use for only one CVD risk factor-MET values. This indicates that the association of depression symptoms with other CVD risk factors generally was as strong in those taking ADMs as in those not taking them. We also found that the association of ADM use with these CVD risk factors was equally strong in those with different levels of depression symptoms. For the one exception (MET values) the association of CVD risk with each depression factor was stronger (more negative) in the relative or absolute absence of the other depression factor. Thus, there was a negative synergy involving a tendency of these factors to suppress or substitute for each other, and their effects were not strictly additive. While we do not have an explanation for why synergy was present only for this one CVD risk factor, nor what might have produced this synergy, the reasons for these findings are likely to become clearer as we better understand the ways that depression symptoms and medicines are related to CVD risk.

Other ancillary analyses also confirmed the robustness of our original models. An analysis using log-transformed BDI scores generated results very similar to our main analysis, as did a test for age interaction with BDI scores and ADM use. Finally, we found that the 
association between SSRI (or any non-TCA or tetracyclic) use and CVD risk factors was essentially the same as the association between all ADMs and CVD risk factors.

Elevated depression symptoms could be a cause, consequence or exacerbating agent for CVD risk. Alternatively, both depression symptoms and CVD risk factors could have a common cause, such as inflammation. There are several plausible explanations for the association between ADM use and CVD risk indicators; while the present study does not allow us to assess their validity, we offer them to stimulate future research to evaluate possible explanations. First, ADM use may be a marker for more severe, chronic or recurrent depression in the past. Second, individuals who qualify as having elevated CVD risk because they take medicines for those conditions might also be more likely to take ADMs, independently of depression status. Third, some side effects of antihypertensive and lipid-lowering medicines (e.g. insomnia, tiredness and impotence) may be identified as symptoms of depression and treated with ADM. Alternatively, as hypothesised, ADMs might increase BP, lipids and BMI. While weight gain is a recognised side effect of most ADMs, little is known about the effects of ADMs on BP and lipids.

\section{Study strengths and limitations}

This is the first study of which we are aware to assess the association between depression symptoms or ADM use and CVD risk in people with type 2 diabetes, and to simultaneously assess the independent association of depression symptoms and ADM use with CVD risk in any population. Other study strengths include the large, multi-ethnic population and the fact that depression symptoms, ADM use and a broad range of cardiovascular risk factors were assessed. Moreover, most CVD risk factors were assessed objectively rather than relying on self-reporting.

The current study also contributes to the growing literature suggesting that negative health outcomes may be associated with depression symptoms across the whole range of depression symptom severity. The fact that few Look AHEAD trial participants had high levels of depression symptoms allowed for a robust analysis of the association between subclinical depression symptom scores and CVD risk factors.

The study also has important limitations. It was not a controlled trial assessing the effects of depression symptoms or ADM use on cardiovascular risk. It was cross-sectional, so we cannot draw conclusions about causal associations between depression measures and CVD risk measures.

Another limitation of the study is the fact that we did not confirm that all patients took ADMs because of depression rather than for other indications, such as smoking cessation, neuropathic pain or other psychiatric conditions, including panic disorder, social anxiety disorder, obsessive-compulsive disorder and post-traumatic stress disorder [23]. Further, we had no information about the dosage or duration of treatment with ADMs. Also, some participants might have failed to disclose that they were taking ADMs, while others who reported that they were not taking ADMs may have discontinued them very recently. However, both of these possibilities would mitigate the likelihood of finding an association between ADM use and CVD risk factors; therefore they would bias the estimated associations toward the null hypothesis.

\section{Conclusions}

In future analyses, we will assess the temporal dynamics of the relationship of ADM use and CVD risk factors in Look AHEAD. Longitudinal studies currently underway, such as the Safety and Efficacy of Sertraline for Depression in Patients with Congestive Heart Failure 
(SADHART-CHF) trial [24], could also help clarify the effects of SSRI, the most commonly prescribed class of ADMs, on cardiac prognosis. Look AHEAD longitudinal studies will also allow us to assess the association of continuous depression symptoms and elevated depression symptoms with health risk factors and negative health outcomes.

\title{
Acknowledgments
}

Funding This study is funded by the National Institutes of Health with additional support from the Centers for Disease Control and Prevention.

\begin{abstract}
This study is supported by the Department of Health and Human Services through the following cooperative agreements from the National Institutes of Health: DK57136, DK57149, DK56990, DK57177, DK57171, DK57151, DK57182, DK57131, DK57002, DK57078, DK57154, DK57178, DK57219, DK57008, DK57135 and DK56992. The following federal agencies have contributed support: National Institute of Diabetes and Digestive and Kidney Diseases; National Heart, Lung, and Blood Institute; National Institute of Nursing Research; National Center on Minority Health and Health Disparities; Office of Research on Women's Health; and the Centers for Disease Control and Prevention. This research was supported in part by the Intramural Research Program of the National Institute of Diabetes and Digestive and Kidney Diseases. The Indian Health Service (IHS) provided personnel, medical oversight and use of facilities. The opinions expressed in this paper are those of the authors and do not necessarily reflect the views of the IHS or other funding sources. Additional support was received from The Johns Hopkins Medical Institutions Bayview General Clinical Research Center (M01-RR-02719); the Massachusetts General Hospital Mallinckrodt General Clinical Research Center (M01-RR-01066); the University of Colorado Health Sciences Center General Clinical Research Center (M01 RR00051) and Clinical Nutrition Research Unit (P30 DK48520); the University of Tennessee at Memphis General Clinical Research Center (M01RR00211-40); the University of Pittsburgh General Clinical Research Center (M01 RR000056 44) and NIH grant (DK 046204); and the University of Washington/VA Puget Sound Health Care System Medical Research Service, Department of Veterans Affairs. The following organisations have committed to make major contributions to Look AHEAD: Federal Express; Health Management Resources; Johnson \& Johnson; LifeScan; OptifastNovartis Nutrition; Roche Pharmaceuticals; Ross Product Division of Abbott Laboratories; Slim-Fast Foods Company; and Unilever.
\end{abstract}

\section{Abbreviations}

ABI

ADM

AHEAD

BDI

CVD

DPP

ILI

MET

SNRI

SSRI

TCA
Ankle-brachial index

Antidepressant medicine

Action for Health in Diabetes

Beck Depression Inventory

Cardiovascular disease

Diabetes Prevention Program

Intensive lifestyle intervention

Metabolic equivalent

Serotonin-noradrenaline reuptake inhibitor

Selective serotonin reuptake inhibitors

Tricyclic antidepressant

\section{References}

1. Egede LE, Nietert PJ, Zheng D. Depression and all-cause and coronary heart disease mortality among adults with and without diabetes. Diab Care. 2005; 28:1339-1345.

2. Egede LE, Zheng D. Independent factors associated with major depressive disorder in a national sample. Diab Care. 2003; 26:104-111. 
3. Lett H, Blumenthal JA, Babyak M, Sherwood A, Strauman T, Robins C. Depression as a risk factor for coronary artery disease: evidence, mechanisms, and treatment. Psychosom Med. 2004; 66:305315. [PubMed: 15184688]

4. Van Melle JP, de Jong P, Spijkerman TA, et al. Prognostic association of depression following myocardial infarction with mortality and cardiovascular events: a meta-analysis. Psychosom Med. 2004; 66:814-822. [PubMed: 15564344]

5. Cohen HW, Gibson G, Alderman MH. Excess risk of myocardial infarction in patients treated with antidepressant medications: association with tricyclic agents. Am J Med. 2000; 108:2-8. [PubMed: 11059434]

6. Taylor CB, Youngblood ME, Catellier D, et al. for the ENRICHD Investigators. Effects of antidepressant medication on morbidity and mortality in depressed patients after myocardial infarction. Arch Gen Psychiatry. 2005; 62:792-798. [PubMed: 15997021]

7. Thombs BD, de Jonge P, Coyne JC, et al. Depression screening and patient outcomes in cardiovascular care: a systematic review. JAMA. 2008; 300:2161-2171. [PubMed: 19001627]

8. Rubin RR, Ma Y, Marrero DG, et al. for the Diabetes Prevention Program Research Group. Elevated depression symptoms, antidepressant medicine use, and risk of developing diabetes during the Diabetes Prevention Program. Diab Care. 2008; 31:420-426.

9. Ryan DH, Espeland MA, et al. Look AHEAD (Action for Health in Diabetes): design and methods for a clinical trial of weight loss for the prevention of cardiovascular disease in type 2 diabetes. Control Clin Trials. 2003; 24:610-628. [PubMed: 14500058]

10. Lange S, Trampisch HJ, Haberi R, et al. Excess 1-year cardiovascular risk in elderly primary care patients with a low ankle-brachial index (ABI) and high homocysteine level. Atherosclerosis. 2005; 178:351-357. [PubMed: 15694945]

11. Ribisl PM, Lang W, Jaramillo SA, et al. for the Look AHEAD Research Group. Exercise capacity and cardiovascular/metabolic characteristics of overweight and obese individuals with type 2 diabetes: the Look AHEAD clinical trial. Diab Care. 2007; 30:2679-2684.

12. The Look AHEAD Research Group. Baseline characteristics of the randomized cohort for the Look AHEAD (Action for Health in Diabetes) Study. Vasc Dis Res. 2006; 3:202-215.

13. Beck, AT.; Steers, RA. Manual of the Beck Depression Inventory. Psychological Corporation; San Antonio, TX: 1993.

14. Rubin RR, Knowler WC, Ma Y, et al. Depression symptoms and antidepressant medicine use in Diabetes Prevention Program participants. Diab Care. 2005; 28:830-837.

15. Norman GR, Sloan JA, Wyrich KW. Interpretation of changes in health-related quality of life: the remarkable universality of a half standard. Med Care. 2003; 41:582-592. [PubMed: 12719681]

16. McDermott MM, Liu K, Criqui MH, et al. Ankle-brachial index and subclinical cardiac and carotid disease: the multi-ethnic study of atherosclerosis. Am J Epidemiol. 2005; 162:33-41. [PubMed: 15961584]

17. American Diabetes Association. Standards of medical care in diabetes. Diab Care. 2005; 28(Suppl 1):S4-S36.

18. Expert Panel on Detection, Evaluation, and Treatment of High Blood Cholesterol in Adults. Executive Summary of the Third Report of the National Cholesterol Education Program (NCEP) Expert Panel on Detection, Evaluation, and Treatment of High Blood Cholesterol in Adults (Adult Treatment Panel III). JAMA. 2001; 285:2486-2497. [PubMed: 11368702]

19. Giorda CB, Avogaro A, Maggini M. for the Diabetes Informatics Study Group. Recurrence of vascular events in patients with type 2 diabetes: epidemiology and risk factors. Diab Care. 2008; 31:2154-2159.

20. Cederholm J, Eeg-Olofsson K, Eliasson B, Zethelius B, Nilsson PM, Gudbjornsdottir S. on behalf of the Swedish National Diabetes Register. Risk prediction of cardiovascular disease in type 2 diabetes: a risk equation from the Swedish National Diabetes Register. Diab Care. 2008; 31:20382043.

21. Gonzalez JS, Safren SA, Cagliero E, et al. Depression, self-care, and medication adherence in type 2 diabetes: relationships across the full range of symptom severity. Diab Care. 2007; 30:22222227. 
22. Penninix BW, Beelman AT, Honig A, et al. Depression and cardiac mortality: results from a community-based longitudinal study. Arch Gen Psychiatry. 2001; 58:229-230. [PubMed: $11231828]$

23. Physicians Desk Reference. 22. Thomson; Montvale, NJ: 2008.

24. Jiang W, O'Connor C, Silva SG, et al. for the SADHART-CF Investigators. Safety and Efficacy of Sertraline for Depression in Patients with CHF (SADHART-CHF): a randomized, double-blind, placebo-controlled trial of sertraline for major depression with congestive heart failure. Am Heart J. 2008; 156:437-444. [PubMed: 18760123] 
Table 1

Depression and ADM use by demographic variables of interest

\begin{tabular}{|c|c|c|c|c|}
\hline Variable & Total $(n)$ & BDI $\geq 11(\%)$ & On ADMs (\%) & BDI $\geq 11$ or on ADMs (\%) \\
\hline Total & 5,145 & $752(14.7)$ & $846(16.5)$ & $1,373(26.8)$ \\
\hline \multicolumn{5}{|l|}{ Age (years) } \\
\hline $45-55$ & 1,621 & $281(17.4)$ & $309(19.1)$ & $507(31.3)$ \\
\hline $56-65$ & 2,650 & $380(14.4)$ & $432(16.3)$ & $698(26.5)$ \\
\hline $66-76$ & 874 & $91(10.5)$ & $105(12.0)$ & $168(19.3)$ \\
\hline$p$ value $^{a}$ & & $<0.0001$ & $<0.0001$ & $<0.0001$ \\
\hline$p$ value $^{b}$ & & 0.0046 & $<0.0001$ & $<0.0001$ \\
\hline \multicolumn{5}{|l|}{ Sex } \\
\hline Male & 2,082 & $218(10.5)$ & $253(12.2)$ & $404(19.4)$ \\
\hline Female & 3,063 & $534(17.5)$ & $593(19.4)$ & $969(31.8)$ \\
\hline$p$ value $^{a}$ & & $<0.0001$ & $<0.0001$ & $<0.0001$ \\
\hline$p$ value $^{b}$ & & 0.001 & $<0.0001$ & $<0.0001$ \\
\hline \multicolumn{5}{|l|}{ Race } \\
\hline African-American/black (not Hispanic) & 803 & $132(16.5)$ & $67(8.3)$ & $174(21.8)$ \\
\hline American Indian/Native American/Alaskan Native & 258 & $70(27.2)$ & $24(9.3)$ & $84(32.7)$ \\
\hline White & 3,253 & $396(12.2)$ & $657(20.2)$ & $893(27.5)$ \\
\hline Hispanic & 681 & $131(19.2)$ & $75(11.0)$ & $183(26.9)$ \\
\hline Asian/Pacific Islander/other/mixed/missing & 150 & $23(15.3)$ & $23(15.3)$ & $39(26.0)$ \\
\hline$p$ value $^{a}$ & & $<0.0001$ & $<0.0001$ & 0.0033 \\
\hline$p$ value $^{b}$ & & $0.008^{c}$ & $<0.0001^{d}$ & $<0.0001^{e}$ \\
\hline \multicolumn{5}{|l|}{ Education } \\
\hline$<13$ years & 1,024 & $211(20.7)$ & $314(13.1)$ & $308(30.7)$ \\
\hline $13-16$ years & 1,915 & $320(16.8)$ & $345(18.0)$ & $562(29.4)$ \\
\hline$>16$ years & 2,094 & $203(9.7)$ & $341(16.3)$ & $468(22.4)$ \\
\hline$p$ value $^{a}$ & & $<0.0001$ & 0.0026 & $<0.0001$ \\
\hline$p$ value $^{b}$ & & $<0.0001$ & 0.0156 & $<0.0001$ \\
\hline \multicolumn{5}{|l|}{${ }^{a}$ value from $\chi^{2}$ test } \\
\hline \multicolumn{5}{|c|}{$b$ value from multivariate logistic regression adjusted for the other three variables in the table } \\
\hline \multicolumn{5}{|c|}{$\begin{array}{l}c \\
\text { Elevated depression symptom scores were more common (test results not shown in table) among American Indian/Native American/Alaskan } \\
\text { Natives than in any other racial/ethnic group (all } p<0.01 \text { ), and elevated BDI scores were more common among African-Americans and Hispanics } \\
\text { than among whites (both } p=0.001 \text { ) }\end{array}$} \\
\hline \multicolumn{5}{|c|}{$\begin{array}{l}d_{\text {ADM use was more common (test results not shown in table) among whites than among African-Americans, Hispanics or American Indian/ }} \\
\text { Native American/Alaskan Natives (all } p<0.001) \text {, and ADM use was more common among Asian/Pacific Islanders than among African-Americans } \\
(p<0.01)\end{array}$} \\
\hline
\end{tabular}




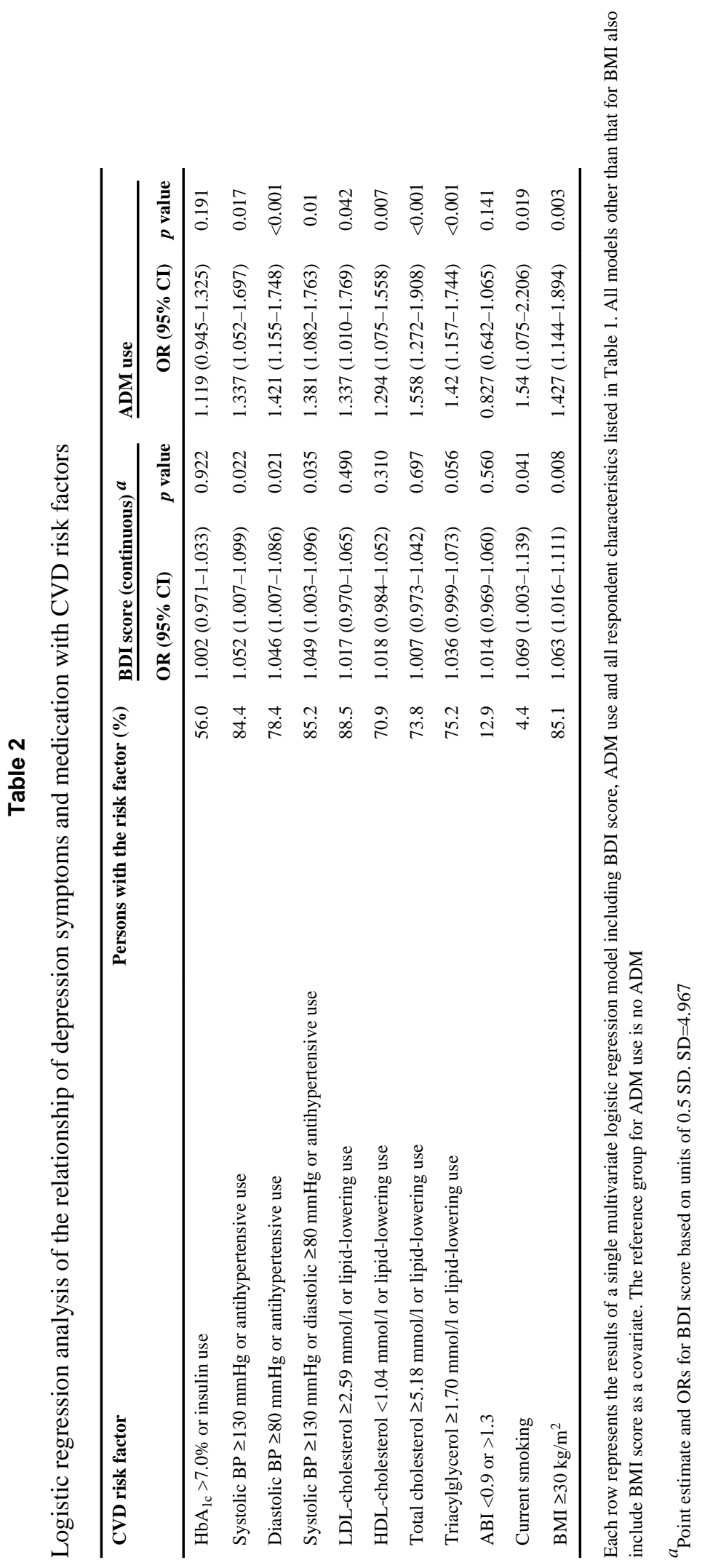

